\title{
Situación actual de la salud sexual y salud reproductiva de los hombres privados de libertad del Centro de Atención Institucional de San José ${ }^{1}$
}

Dorita Rivas Fonseca ${ }^{2}$ Adriana Ortiz Obando ${ }^{3}$

Institución: Posgrado en enfermería ginecológica, obstétrica y perinatal

Centro de Atención Institucional de San José (CAI)

\section{COMO CITAR}

Ortiz, A. y Rivas, D. (Octubre, 2013). Situación actual de la salud sexual y salud reproductiva de los hombres privados de libertad del Centro de Atención Institucional de San José. Rev. Enfermería Actual de Costa Rica, 25, 1-15. Recuperado de:: <http://www.revenf.ucr.ac.cr/sexual.pdf> ISSN 1409-4568

\section{RESUMEN}

El objetivo de esta investigación fue conocer la situación actual respecto del tema de salud sexual y salud reproductiva de los privados de libertad del Centro de Atención Institucional (CAI) de San José. Es un estudio descriptivo y transversal. Mediante un muestreo estrategio se determinó la participación de 102 hombres. Para obtener información se aplicó un cuestionario autoadministrado con preguntas cerradas y abiertas. Como resultado relevante respecto del perfil sociodemográfico, se evidenció que los privados de libertad es un grupo muy heterogéneo. En cuanto a la salud sexual y salud reproductiva, ellos relacionan el primer concepto con la prevención de enfermedades y el segundo con la reproducción, lo cual señala las limitaciones en cuanto al conocimiento en los temas, aspecto que influye en las actividades de su vida diaria y el autocuidado. Se concluye que la investigación por parte de enfermería ginecoobstétrica en poblaciones privadas de libertad es casi nula no solo en el país, sino en el mundo, máxime si se trata con población masculina. En el caso del Centro Penitenciario CAI la atención en salud no es suficiente para la cantidad de privados de libertad que habitan (sobrepoblación de casi un 50\%), ello implica un deterioro de las condiciones físicas y de salud de estas personas, así como de su salud sexual y reproductiva.

Palabras clave: hombres-privados-de-libertad, salud-sexual, salud-reproductiva.

\footnotetext{
${ }^{1}$ Fecha de recepción: 17 de abril 2013

Fecha de aceptación: 23 de julio 2013

2 Enfermera. Centro Médico Santa Mónica. Correo electrónico: doririvas@gmail.com

${ }^{3}$ Enfermera. Servicios profesionales. Correo electrónico: arianita.ortiz@hotmail.com
} 


\title{
Current situation of sexual and reproductive health of men deprived of liberty in the Institutional Care Center of San Jose ${ }^{1}$
}

Institution: Postgraduate gynecological, obstetric and perinatal nursing

Dorita Rivas Fonseca ${ }^{2}$ Adriana Ortiz Obando ${ }^{3}$ Institutional Care Center of San Jose (CAI)

\section{CITED}

\begin{abstract}
The objective of this research was to determine the current status of the issue of sexual and reproductive health of the prisoners Institutional Care Center (CAI) of San Jose. It is a descriptive study. Through a strategic sampling determined the participation of 102 men. The information was obtained by applying a self-administered questionnaire with closed and open questions. As a result relevant to your socio-demographic profile, it appears that deprived of their liberty is a very heterogeneous group. As regards sexual and reproductive health, the first concept they relate to the prevention of disease and the second reproductive aspects, this shows limitations in knowledge on the topics, something that affects the daily life activities and self-care. It is concluded that research by nurses Gyneco-obstetric in the deprived of liberty is almost null not only in the country but in the world, especially if it comes with the male population. In the case of CAI Prison, health care is not enough for the number of inmates who inhabit (overpopulation of almost 50\%), this implies a deterioration in health and physical condition of these people, as well as sexual and reproductive health.
\end{abstract}

Keywords: prisoners-men, reproductive health, sexual health.

\footnotetext{
${ }^{1}$ Date of receipt: april 17, 2013

Date of acceptance: july 23, 2013

${ }^{2}$ Nurse. Centro Médico Santa Mónica. E-mail: doririvas@gmail.com

${ }^{3}$ Nurse. Professional services. E-mail: arianita.ortiz@hotmail.com
} 


\section{INTRODUCCIÓN}

La presente investigación pretende informar acerca de la realidad en el tema de la salud sexual y salud reproductiva de un grupo de hombres privados de libertad de un centro penitenciario de nuestro país. La población de personas sometidas al control y la atención institucional ha aumentado: según el Ministerio de Justicia y Paz (2012), específicamente el Centro de Atención Institucional (CAI) San José, se registra más de un $61 \%$ de sobrepoblación carcelaria en el mes de abril, 2012. (p.14). Sin embargo, aun cuando se confirma la sobrepoblación carcelaria, se desconocen las condiciones y la atención de la salud en las cárceles, dado que representa una población minoritaria, marginada y estigmatizada. Debido a lo anterior, la cárcel es considerada un escenario, cuya población es más vulnerable de sufrir problemas de salud.

La salud no establece distinciones entre seres humanos, dado que es inherente a todo grupo del cual los privados de libertad son parte; por ende, su condición no les niega el derecho a demandar servicios de salud que garanticen, de alguna forma, el respeto a su dignidad y a su integridad física, mental y emocional.

Debido a lo anterior; el siguiente trabajo se aboca a develar la realidad que viven los individuos en este entorno con el fin de ofrecer un panorama general acerca de la salud sexual y reproductiva y generar investigaciones en pro de la salud integral de los privados de libertad.

Esta investigación se llevó a cabo en el Centro de Atención Institucional de San José (CAI). Aunque sí se ha estudiado el tema, las investigaciones se centran solo en el sector femenino, aparte de que se enfatizan temas como enfermedades de transmisión sexual, consumo de drogas, y otros tópicos relacionados con los patrones masculinos hegemónicos; sin embargo, no se ha abordado el tema de manera integral y, muchos menos, se enfatizan las necesidades de la población en estudio. Tales carencias refuerzan la idea de fortalecer los conocimientos, responder a las dudas que manifiesten los privados de libertad en materia de salud sexual y reproductiva y ofrecer estrategias adecuadas de intervención.

Por último, puesto que la población de estudio no ha participado lo suficiente en investigaciones nacionales respecto del tema de salud, es imprescindible instar a los profesionales de enfermería ginecológica, obstétrica y perinatal para que ofrezcan, desde su ámbito, un abordaje integral, universal, oportuno y eficaz.

Respecto de su objetivo, el propósito general de la investigación fue describir la situación de salud sexual y reproductiva en la población mencionada.

\section{MATERIALES Y MÉTODOS}

El presente estudio corresponde a una investigación aplicada, definida por Vargas (2009), citando a Murillo (2008), como “investigación práctica o empírica”. Este tipo de estudio se caracteriza por,

la aplicación de los conocimientos adquiridos, a la vez que se adquieren otros, después de implementar y sistematizar la práctica basada en investigación, además, del uso del 
conocimiento y los resultados de investigación dan resultado a una forma rigurosa, organizada y sistemática de conocer la realidad (p.159).

El tipo de investigación es descriptiva y transversal. Para la muestra se eligieron 102 hombres privados de libertad, escogidos con un muestreo estratégico.

Para la recolección de datos, se aplicó un cuestionario autoadministrado individual, el cual, según Hernández et al. (2007), “...se proporciona directamente a los participantes (...) se entrega al participante y este lo responde." (p.p. 235-236). El cuestionario incluye preguntas abiertas, debido a que “...no delimitan de antemano las alternativas de respuesta." (Barrantes, 2000, p. 199), y cerradas, las cuales “...contienen categorías o alternativas de respuesta..." (Barrantes, 2000, p.196) acerca de la salud sexual y reproductiva .

El cuestionario constó de cuatro partes: la primera abarcó preguntas relacionadas con características personales como edad, estado civil y delito cometido, entre otras; la segunda parte, constó de preguntas acerca de salud sexual (conceptos, prácticas, disfunciones, entre otras); la tercera incluyó preguntas respecto de la salud reproductiva (conceptos, paternidad, métodos anticonceptivos, ITS, entre otros aspectos) y la cuarta parte, sobre mitos de sexualidad, constó de un cuadro con siete proposiciones en las que el privado de libertad marcaba verdad o mentira de acuerdo con su opinión al respecto.

Se negociaron alrededor de cinco horas para que el grupo investigador aplicara los cuestionarios. Para llevar a cabo el trabajo de campo, se utilizaron las instalaciones facilitadas por el centro penitenciario, constituidas por tres aulas unidas, en donde regularmente se imparten lecciones a los privados de libertad. Cada grupo, integrado por 20 personas aproximadamente, respondió al cuestionario en un lapso de treinta a cuarenta y cinco minutos.

\section{Consideraciones éticas.}

Como en toda investigación, es obligatorio practicar los principios bioéticos, los cuales incluyen la autonomía, a partir de la que las personas son "...constructoras de un imperativo moral que establecen como ley universal de cumplimiento obligatorio." (Leiva, 2010, p.9); lo anterior significa que cada persona es un ser autónomo que actuará de acuerdo con sus ideales morales y sin ser coaccionado, dado que cada participante decidió si quería participar en el estudio.

Otro principio es la justicia, definida por la misma autora como “...el tratamiento igualitario a todas las personas." (p. 8); por tanto, la situación de privados de libertad correspondiente a la población en estudio no fue motivo para tratarlos de manera distinta. Por último, el principio de beneficencia se entiende como "...contribuir positivamente a su bienestar." y el de no maleficencia, como "...no hacer daño..." (Leiva, 2010, p.9); ambos se interrelacionan $\mathrm{y}$ son fundamentales en cada investigación, dado que su cumplimiento garantiza que los participantes no sufran ningún tipo de daño; por el contrario, la idea es que la población obtenga una enseñanza positiva o conocimiento a partir del trabajo. Dentro las consideraciones éticas se destaca el consentimiento informado como parte fundamental de cualquier investigación, puesto que es parte del respeto a la autonomía, principio que se reforzó durante la Revolución Francesa y que se aplicó en el estudio. 


\section{RESULTADOS}

\section{Perfil sociodemográfico.}

Respecto de sus edades, el grupo es bastante heterogéneo: hay privados de libertad con edades que abarcan desde los 19 años hasta los 60 años; en primer lugar, la edad de la mayor cantidad de privados de libertad comprende entre los 29 a 38 (38,23\%), luego, está el rango comprendido entre los 19- 28 años de edad (32,35\%); en tercer lugar están quienes poseen una edad superior a los 59 años; los rangos con los mayores porcentajes revelan que la población en estudio se encuentra en una edad productiva.

En cuanto a la nacionalidad, el $69,60 \%$ de los privados de libertad encuestados son costarricenses y el 28,43\% restante incluye extranjeros, entre cuyos países de origen se encuentran Nicaragua, Colombia, México, Cuba, Guatemala, El Salvador, Rumania, Estados Unidos y Canadá.

En relación con la escolaridad, el 33,33\% señaló haber cursado secundaria incompleta como su nivel educativo, mientras que un $19.60 \%$ cuenta solo con primaria incompleta. El nivel universitario tanto completo como incompleto registró un $10,78 \%$ cada uno, cifra representativa a partir de la que se deduce que haber cursado la educación superior y formal no exime a una persona en cuanto a cometer un delito.

Respecto del lugar de residencia antes de vivir en la cárcel, un 60,78\% de los privados de libertad residía en San José en los llamados "barrios del Sur", ya sea en Desamparados, Hatillo u otros lugares aledaños y un 9,80\% habitaba en Heredia y Alajuela. Entre los encuestados había un indigente y cinco personas que no respondieron a la pregunta.

En lo que respecta al estado civil, el 29,41\% se refiere a los solteros, el 27,45\% vive en unión libre y el $23,53 \%$ representa el porcentaje de quienes están casados.

Los oficios antes de ser encarcelados, se registraron 62 tipos de trabajos que incluyen desde médicos, saloneros, periodistas, agricultores, contadores, entre otros; aun así, tres oficios obtuvieron los mayores porcentajes: Comerciante: $8,82 \%$; Oficial de seguridad: 6,86\% y Construcción: 5,88\%.

Entre los motivos por los cuales los hombres se encuentran privados de libertad está haber cometido robo de vehículos o agravado (30,39\%); luego está el delito de las "drogas" (16,67\%), en el que se incluye el tráfico y venta de drogas. El delito de homicidio presentó igual porcentaje $(16,67 \%)$ y comprende tanto homicidio simple, como tentativa de homicidio. En el caso del delito "violencia", corresponde a violencia física, sexual y doméstica.

Dentro de la condición legal en la que se encuentran en el centro penitenciario se obtuvo lo siguiente: debido a que el centro penitenciario es de "paso", un 55,88\% se encuentra indiciado, concepto que, según la Dirección General de Adaptación Social (2007), se entiende como “...las personas privadas de libertad que se encuentran a la orden de una autoridad jurisdiccional por tener un proceso penal pendiente, en el cual se les atribuye la responsabilidad por la comisión de un determinado delito, es decir, figuran como imputados." (p.9). E1 $37,25 \%$ está sentenciado; es decir, son personas puestas a la disposición de una entidad correccional para cumplir 


\section{Revista Electrónica Enfermería Actual en costa Rica}

una sentencia condenatoria impuesta por una autoridad jurisdiccional, como lo establece esta misma dirección; por último, un $6.86 \%$ no brindó información al respecto.

Además, a los sentenciados se les interrogó acerca del tiempo de sentencia, el cual es muy variado: va desde los 3 años hasta los 140 años. Al final, se indagó sobre el tiempo que ha transcurrido desde que viven en el centro penitenciario: algunos tienen entre siete y 29 meses de permanecer allí; sin embargo, la mayoría ha habitado el lugar entre uno y siete meses.

\section{Salud sexual.}

La salud sexual de un ser humano abarca un gran panorama de temas que trascienden las prácticas sexuales, ya sea en pareja o como individuo.

Con el fin de investigar cuál es el concepto que los privados de libertad conocen acerca del término "salud sexual", se planteó una pregunta abierta, cuyas respuestas fueron variadas; sin embargo, la información más significativa se muestra a continuación:

- "El cuidado y la forma de tener nuestro cuerpo física y mentalmente bien, sano para llegar a estar bien con uno mismo y con nuestra sexualidad". (Informante 10)

- "Protegerse de enfermedades". (Informante 3)

- "Higiene". (Informante 9)

-"Sexo". (Informante 1)

-"No sé nada, nunca me han hablado de eso". (Informante 13)

Un 63,72\% respondió que sí tiene pareja en la actualidad, de los cuales un 75,38\% tiene una pareja; un $6,15 \%$, dos parejas y un 3,08\%, tiene tres parejas. Por otro lado, un 36,27\% contestó que no tiene pareja. De ese $63,72 \%$ que tiene pareja, no todos son visitados. Un 30,77\% de los privados de libertad con pareja recibe una visita íntima establecida, un $35,38 \%$ está en proceso de aprobación esa visita íntima y un $18,46 \%$ no tiene ni está en aprobación.

Dentro de las prácticas sexuales que realizan con su pareja se obtuvo que un $80,39 \%$ practica besos y caricias con su pareja; un $72,55 \%$ practica la relación sexual coital; un $67.65 \%$, la relación sexual oral; un $39,21 \%$, la masturbación mutua y un 16,69\%, la masturbación individual.

La autocaricia es común en un 65,69\% de los privados de libertad; un 43,28\% la práctica entre una y dos veces por semana; un 22,39\% cada 15 días, un 20,89\%, cada mes, y un $11,94 \%$, todos los días.

Se elaboraron dos preguntas abiertas acerca de si consideran que sus prácticas sexuales son placenteras: un 92,16\% respondió que sus prácticas sexuales sí son placenteras, lo cual respaldaron con argumentos como los siguientes:

- "Porque ambos quedamos satisfechos". (Informante 2)

- "Solo el hecho de estar con la persona que uno ama". (Informante 6)

- "Trato de que sea placentero". (Informante 31) 


\section{Revista Electrónica Enfermería Actual en costa Rica}

Por otro lado, una parte de la población piensa que sus prácticas son placenteras por otras razones como:

- "Porque a mí me gusta". (Informante 12)

- "Por lo menos yo quedo complacido". (Informante 46)

- "El resultado siempre fue el agrado de estar y compartir con mi compañera". (Informante 5)

- "Desestrés muy grande". (Informante 29)

Para la segunda pregunta respecto de si para ellos es relevante satisfacer a su pareja, un 95,10\% respondió que sí; no obstante, las respuestas varían entre sí:

- "Lo disfrutamos ambos". (Informante 3)

- "El placer de satisfacerla aumenta mi propio placer y bienestar". (Informante 22)

Entre otras razones, los entrevistaron agregaron:

- "Por quedar bien en la relación". (Informante 8)

- "Si no lo está, fijo me cambia". (Informante 46)

- "Si no viene el rechazo". (Informante 59)

- "Es derecho de ella". (Informante 37)

- "Por el problema de la infidelidad". (Informante 87)

De lo anterior, se infiere que sienten miedo a ser rechazados o víctimas de infidelidad por parte de sus parejas.

Luego, se les preguntó acerca del número de personas con las que han mantenido relaciones sexuales coitales, orales y anales en los últimos 6 meses: aunque la mayoría oscila entre una y dos personas, un entrevistado respondió haber estado con 10 personas en los últimos 6 meses (esta persona obviamente no ha permanecido mucho tiempo en prisión), 15 no respondieron y un alto porcentaje de hombres son abstemios.

En cuanto al logro de orgasmos en las prácticas sexuales, un 70,59\% los experimenta en todas sus relaciones sexuales; un $19,61 \%$, a veces; el $2,94 \%$, nunca y un $6,86 \%$ no respondió a esta pregunta. En relación con el tema es imprescindible considerar que, desde la construcción social, la sexualidad del hombre se ha limitado a la erección, penetración y eyaculación; por ende, cuando no se encuentra en condiciones de tener relaciones sexuales coitales, es tachado de débil o de enfermo e, incluso, se cuestiona su hombría. Considerando que la sexualidad es integral, no se puede juzgar el desempeño sexual de un hombre a partir del número de eyaculaciones y penetraciones que concrete, dado que su ánimo también se ve afectado por circunstancias diversas, al igual que la mujer.

El tema anterior se relaciona con el de las disfunciones sexuales: más del 50\% de los participantes nunca ha padecido de ninguna de las disfunciones sexuales acerca de las que se les preguntó; sin embargo, un 10,78\% alguna vez ha tenido dificultad para la erección y un $19,61 \%$ ha tenido eyaculación precoz. Las disfunciones sexuales no encajan dentro de lo que es ser hombre dado que, según Campos y Salas (2002), “...la demostración 
constante de la virilidad se concretiza en estar siempre listo para tener sexo, siempre con el pene erecto, que "funciona" siempre durando mucho para eyacular." (p.25).

\section{Salud reproductiva.}

La salud reproductiva constituye un elemento necesario de explorar en este grupo de hombres, dado que es una forma en la que los privados de libertad desarrollen potencialidades vinculadas con el cuidado de sus cuerpos y las prácticas sexuales seguras y responsables; por ende, se consideró relevante que contestaran a la pregunta ¿qué entiende usted por salud reproductiva? Al respecto las respuestas fueron variadas:

- "Ser responsable para nuestra reproducción". (Informante 2)

- "Tener hijos sin dificultad". (Informante 10)

- "Conjunto de información, práctica y atención de las diversas fases de la sexualidad que comprende específicamente el área de reproducción”. (Informante 23)

-"Es muy importante la relación sexual en la vida de las personas para un buen embarazo". (Informante 3)

- "Cuidar mi salud". (Informante 15)

-“Saber cuántos hijos puedo tener, según mi estatus económico”. (Informante 65)

- "Saber usar el condón”. (Informante 100)

-“Tener hijos en pareja responsablemente y sin vicios”. (Informante 18)

- "No ser estéril". (Informante 70)

En cuanto a cuáles métodos de planificación familiar conocen, un 74,51\% conoce acerca del 1 condón, método en el que el hombre tiene la responsabilidad primaria, dado que es el más conocido y el que más usan. Un $2,94 \%$ se refirió al gel espermicida y, en menor cantidad, a la abstinencia. Respecto de la frecuencia con la que los utilizan, un $37,14 \%$ respondió que siempre; un $20 \%$, casi siempre y un $34,28 \%$, a veces.

Continuando con el tema de planificación familiar, se indagó acerca de quién escoge el método anticonceptivo: 70,56\% lo decide junto con su pareja; en un 11,76\%, solo lo escoge la pareja; en un 2,94\%, es responsabilidad del entrevistado y un $12,74 \%$ no respondió a esta pregunta.

En una de las preguntas abiertas se abordó el tema de si consideran que, al menos, cada año, el profesional en salud les examine las mamas, el pene, los testículos y la próstata. En resumen el 92.16\% respondió de manera positiva; sus comentarios fueron:

- “Sí, por alguna enfermedad, ya sea cáncer o de transmisión sexual”. (Informante 2)

- "Para darnos cuenta si estamos bien de salud y nuestros órganos sexuales". (Informante 4)

- "Es un derecho del ser humano". (Informante 85)

- "Para tener relaciones sexuales seguras y sanas". (Informante 60)

- "Pero solo por una profesional mujer". (Informante 25)

El 3,92\% respondió que no creen necesario ese chequeo anual y esa misma cantidad no respondió a la pregunta. 
Luego, se indagó respecto de si ellos se realizan el autoexamen de mamas y testículos: sus respuestas fueron variadas: el 56,86\% respondió que sí se autoexaminan las mamas y los testículos, mientras que el 39,21\% restante contesto que no. Aunque hay un sector que practica el autoexamen, no fue posible saber si lo hacen de la manera correcta, dado que es un diagnóstico situacional; no obstante, la espera es que se elaboren más estudios relacionados con el tema.

Se les interrogó también en cuanto a la educación sexual que les brinda el centro penitenciario: un 1,96\% respondió que sí le ha brindado educación, pero no ahondó acerca de qué temas; el un 90,20\% afirmó que no se le ha proporcionado información alguna y un 7,84\% no respondió.

En cuanto a las infecciones de transmisión sexual (ITS), se preguntó si alguno las ha padecido en los últimos 6 meses: cuatro de los participantes de la investigación (3.92\%) respondieron que sí, pero solo uno especificó la enfermedad (papiloma); el 91,18\% respondió negativamente.Aun cuando solo cuatro personas afirmaron padecer alguna ITS en los últimos 6 meses, la cantidad es relevante, máxime si se considera que las ITS son de las principales consecuencias de una relación sexual, ya sea coital, anal u oral; sin protección con un método de barrera: “...las ITS pueden ser transmitidas entre dos personas cualquiera independientemente del sexo o la edad- por sexo vaginal, anal, y oral y por contacto piel a piel durante el sexo." (Engender Health, 2002, p.51).

Se les preguntó además la rapidez con la que el centro penitenciario los atiende en caso de presentar alguna enfermedad: un 58,82\% respondió que, en caso de padecer alguna ETS, no creen tener acceso al servicio de salud, el $21,57 \%$ respondió que a veces; las opciones siempre y casi siempre obtuvieron un 3,92\% cada una y un $11,76 \%$ no respondió a esta pregunta.

También, se les interrogó acerca de cómo es la atención por parte del servicio de salud del centro penitenciario: un 61,74\% respondió que el servicio de salud es malo; un 20,59\%, que es regular; un 5,88\%, que es bueno; un 2,94\% que es excelente; un $1,96 \%$, que es muy bueno y el 6,86\% no respondió a esta pregunta.

Por último, se les solicitó algunas sugerencias, entre las que se destacan:

- “Que sean más eficientes en la salud”. (Informante 8)

- "Charlas informativas". (Informante 2)

- "Que sean más humanos". (Informante 5)

- "Valorar el ingreso de otro doctor, dado que llevan tres meses con un solo médico para más de 1000 presos". (Informante 48)

- "Más personal e infraestructura". (Informante 38)

- "Valoración médica de nuestra sexualidad". (Informante 10)

- "Mejor trámite y horario de visita íntima". (Informante 72)

- “Atención especializada". (Informante 44)

- "Dentro de los oficiales que haya un enfermero". (Informante 65)

- "Que el médico dé todas las medicinas". (Informante 43)

- "Sigan adelante, que no se quede en la investigación nada más, sino que sea una semilla para que germinen los centros penales, donde la atención en salud es una carencia muy notable”. (Informante 23) 


\section{DISCUSIÓN}

Más del 50\% de los privados de libertad encuestados cuenta con una familia, con o sin hijos, en la que la mayoría fungen como proveedores, rol que se destaca dentro de la construcción de la masculinidad que, en muchas ocasiones, al no poder solventar esas necesidades se ven obligados a cometer delitos penados por la ley.

Tal como lo expone el INAMU (2002),

La ideología patriarcal ha asignado como mandato masculino la generación de ingresos y la proveeduría. Efectivamente, mientras que la proveeduría por una parte se constituye en mandato que configura la identidad masculina y paterna, por otra parte, y al mismo tiempo, contiene en su misma constitución, elementos que conducen a la evasión de esta función. La proveeduría tiene un fuerte peso simbólico como función netamente masculina (lo cual es referente tanto para hombres como para mujeres), a pesar de que no necesariamente todos los hombres la cumplen y que, cada vez más, las mujeres tienden a asumirla parcial o totalmente. Además, es necesario tomar en cuenta que la proveeduría, más allá de cualquier definición, se constituye en un deber de los adultos $\mathrm{y}$, por lo tanto, en un derecho social y económico de la niñez, imprescindible para cumplir con su adecuada protección. (Carcedo y Sagot, p.17-18).

Campos y Salas (2002) se refieren a los mandatos sociales, los cuales abarcan “...el éxito en el trabajo, en lo económico y en cuanto a desafiar el peligro de manera permanente.” (p.25), razón por la que, debido a la presión social, los hombres se prestan para infringir la ley. En la mayoría de casos el delito que más se comete es el robo para el que grupos integrados por diversos individuos se unen para robar y, de esta manera, mejorar su calidad de vida y convertirse en proveedores y figuras de poder. En principio, los delitos son "sencillos", mas luego aumentan en gravedad, lo cual se consideran una vía para recalcar la dominación y la imposición masculina y establecer relaciones violentas que afectan no solo sus vínculos delictivos, sino también su esfera de vida privada.

Respecto de la salud sexual, la mayor parte de la muestra de privados de libertad encuestados relaciona el concepto de salud sexual con la prevención de enfermedades exclusivamente; no obstante, a pesar de que dicho tema es un componente esencial, la definición que brinda la OMS aclara que no se trata solo de la ausencia de enfermedades o dolencias en todos los aspectos relacionados con el sistema reproductivo y sus funciones y procesos, por el contrario, la OMS (s.f.) considera que es un estado general de bienestar físico, mental y social y de disfrute sin riesgo. De lo anterior se rescata que muchos de los entrevistados cuentan con una visión integral en materia de salud sexual, dado que incluyen temas como las formas de cuidar el cuerpo y la mente, la comunicación y el respeto hacia la pareja, métodos de planificación y sexualidad responsable.

Briceño y Chacón (2001) mencionan que el hombre debe ser mujeriego, puesto que cuántas más mujeres tenga, más hombre es; desde esa perspectiva, es comprensible por qué algunos entrevistados tienen más de una pareja, aun cuando el reglamento de la visita íntima establece que solo se tramita con una de las parejas. 
La visita íntima implica todo un proceso administrativo, tal como se establece en el Reglamento Técnico del Sistema Penitenciario, decretado en el año 2007, el cual decreta que los encuentros serán una vez cada 15 días, en un cuarto apropiado, por un lapso de cuatro horas. Los derechos en salud sexual disponen que toda persona tiene derecho al disfrute pleno de su sexualidad, aspecto fundamental para su salud y bienestar físico, psicológico y social; sin embargo, la visita íntima restringe este derecho de todos los privados de libertad en nuestro país, porque no pueden establecer contacto íntimo con su pareja ( o con la persona elegida para tal fin) más allá de lo permitido, lo cual contraría el derecho a gozar de autonomía, lo cual redunda en una imposición de carácter coercitivo.

Según la OMS (2006), la sexualidad se vivencia y se expresa a través de pensamientos, fantasías, deseos, creencias, actitudes, valores, conductas, prácticas, papeles y relaciones interpersonales, por lo que las prácticas sexuales dependen de la pareja y del individuo en sí y de lo que ambos quieran; a pesar de ello, como se ha discutido en otras preguntas, esa libertad en las prácticas sexuales es restringida no solo por el espacio físico, sino porque debe desarrollarse en un lapso de cuatro horas, cada quince días, limitación que atenta contra la integridad de los participantes.

La autocaricia es una “....actividad placentera que puede aliviar el estrés, brinda al individuo una sensación de bienestar y mejora el sueño y el estado de ánimo, también puede aliviar la tensión sexual en momentos cuando el compañero sexual no está disponible, interesado o deseoso." (Engender Health, 2008, p.311). Como se dijo en su momento, el CAI excede su capacidad; no obstante, los hombres privados de libertad han encontrado la manera de vivir su sexualidad individualmente por medio de la masturbación. La Asociación Mundial de Sexología, citada por Guezmez (2004, p. 37), expresó que “...el desarrollo pleno de la sexualidad es esencial para el bienestar individual, interpersonal y social”, lo cual significa que al verse restringidos por su condición, ellos ejercen su derecho a la sexualidad por medio de esta práctica que pueden disfrutar derecho siempre y cuando no violen el espacio físico ni personal de los otros privados de libertad con los que conviven.

La gran mayoría de privados de libertad consideran que sus relaciones sexuales son placenteras, algunos mencionan "la satisfacción mutua", la cual se relaciona con la Cuarta Conferencia Mundial Sobre la Mujer (CCMM). En el capítulo de salud sexual y derechos reproductivos que concierne a las relaciones igualitarias entre la mujer y el hombre respecto de las relaciones sexuales y la reproducción, conceptos que perciben como distintos, dado que se tiene derecho al placer y a la procreación, aunque una no dependa de la otra respecto de vivir la sexualidad de manera plena.

A pesar de que más del $50 \%$ de los entrevistados refieren no haber tenido problemas de disfunción sexual, cabe resaltar que un $19,61 \%$ sí reporta casos de eyaculación precoz y un $10,78 \%$ ha presentado dificultad para la erección; entre otras complicaciones “...se detectan niveles más bajos de calidad de vida, cardiopatías, hipertensión, diabetes, índices elevados de enojo y depresión" (OMS, OPS, WAS, 2000, p.16). Respecto de lo mencionado, es imprescindible señalar que la población no solo necesita fármacos para tratar sus padecimientos, sino también psicoterapia y terapias sexuales breves de pareja, tratamientos que no se suelen ofrecer a la población en estudio.

Respecto de la salud reproductiva, la Organización Mundial de la Salud (OMS), citada por The Lancet (1994) define la salud reproductiva de la siguiente manera: 
La salud reproductiva es un estado general de bienestar físico, mental y social, y no de mera ausencia de enfermedades o dolencias, en todos los aspectos relacionados con el sistema reproductivo y sus funciones y procesos. En consecuencia, la salud reproductiva entraña la capacidad de disfrutar de una vida sexual satisfactoria y sin riesgos de procrear, y la libertad para decidir hacerlo o no hacerlo, cuándo y con qué frecuencia. (p.1)

Las respuestas de los privados de libertad se centran en la reproducción; es decir, no abarcan el tema de manera integral como lo establece la OMS.

Tomando en cuenta que las principales consecuencias de una relación sexual, ya sea coital, anal o rectal, sin protección de un "método de barrera", se refieren a las ITS y a los embarazos no deseados: en este caso, más del $50 \%$ de esa población comentó que a veces o casi siempre los usan, lo cual genera un mayor riesgo.

En esta población, pese a ser una muestra de hombres y en condición de privados de libertad, se infiere una inclinación positiva en cuanto a condiciones de igualdad entre hombres y mujeres, en cuanto a controlar las cuestiones relativas a su sexualidad.

La anticoncepción representa una responsabilidad conjunta de la pareja; por ende, “...el método que se escoge debe reflejar las necesidades y las preocupaciones de la pareja. Aun cuando lo ideal es que ambos participen en la elección del método anticonceptivo adecuado, lo cierto es que su uso recae en tan solo un miembro de la pareja; sin embargo, si la pareja elige un método femenino, como anticonceptivos orales o implantes Norplant, el hombre desempeña un papel relevante tanto en su uso, como en su efectividad." (Engender Health, 2008, p. 17).

Tras un análisis de las respuestas obtenidas a partir del instrumento, es notable que en los entrevistados hay una ruptura respecto de la construcción hegemónica de la masculinidad, puesto que se detectó una población con mayor conciencia y valorización personal, la cual resulta de una mayor madurez psicológica y social; es decir, el hombre aprende valores y comportamientos modificables; por ende, se afirma que el género, a diferencia del sexo, es transformable.

Por otro lado, parte del derecho a la información y la educación sexual implica que deben estar libres de prejuicios para fomentar la toma de decisiones libres e informadas, así como la igualdad de oportunidades y la equidad. Las personas tienen además derecho a ser educadas y preparadas para asumir con responsabilidad las consecuencias de su conducta sexual y reproductiva.

En opinión de Campos y Salas (2002), una persona puede vivir su sexualidad de manera integral y responsable si dispone de información amplia, objetiva, científica, con diversos enfoques acerca de su sexualidad; si tiene la oportunidad de expresar y responder a sus dudas, además de reflexionar sobre sí y sobre su vida afectiva. Aparte, el sujeto debe ser capaz de disfrutar sin hacer daño físico y psicológico, ni a sí ni a otros, de experimentar placer con responsabilidad, de respetar valores universales de convivencia y de ser asertivo en cuanto a reconocer y defender sus derechos y los derechos de los otros. La enseñanza es conocer cómo es mi cuerpo y cómo debería ser. 
Según la OMS (2008), “...las infecciones de transmisión sexual son causas importantes de lesión en las trompas de Falopio y de infertilidad en la mujer. Un 30\% a un $40 \%$ de los casos de infertilidad femenina obedecen a una lesión de las trompas de Falopio posterior a una infección.” (p.15). Al respecto, un estudio en Belice en el año 2009, demuestra que la prevalencia de VIH en prisiones es mayor que la población en general; por ende, dichos espacios son vistos como reservorios de esa enfermedad.

En cuanto al servicio que reciben, los privados de libertad lo calificaron como malo, al cual se aúna la carencia de información y acceso a métodos seguros, eficaces, asequibles y aceptables de planificación de la familia; así de servicios adecuados para la atención de la salud. En este sentido, los entrevistados sugirieron mejor abordaje de sus derechos en cuanto a información y educación sexual; es decir, datos completos, verídicos y con sustento científico actualizado.

En nuestra cultura existe una gran cantidad de mitos y creencias respecto de la sexualidad, los cuales se han instalado en la mente de muchas personas, al punto de generarles miedos, dificultades y trabas, en lugar de contribuir a una salud sexual y reproductiva sana e integral.

En relación con los roles de género, la mayoría demostró su preferencia por la equidad; los roles se refieren a las expectativas, a las conductas permitidas y prohibidas y a los comportamientos apropiados e inapropiados. La estructura social prescribe las acciones de cada género y las presenta como si fueran inherentes; por ejemplo, en cada cultura se pauta las expectativas del género masculino y del femenino, además de se les atribuyen roles, atribuciones y estereotipos.

En el caso particular de este estudio, las respuestas distan de la teoría ofrecida por Campos y Salas (2002), para quienes la virilidad se caracteriza por estar, siempre listo para tener sexo; siempre con el pene erecto, que "funciona"; siempre durando mucho para eyacular; siempre dando satisfacción sexual a las mujeres (como nadie lo hace).

Según Cantera (2001), el análisis de género permite establecer diferencias a partir de las que se infiere una nueva práctica de valores y comportamientos en los denominados "masculinos", no tanto por su naturaleza, sino por un proceso de socialización. Si se pensara que todos los hombres actúan de acuerdo con el modelo, la convivencia estaría dominada por una visión esquemática del mundo en el cual no habría valores positivos y en el que las mujeres padecerían una explotación y subordinación constantes.

Cada hombre desarrolla su propio carácter y personalidad, según sus propias experiencias de vida y de acuerdo con las influencias que ejerce sobre él su entorno social (la familia, el vecindario, la escuela, la iglesia, entre otros). Dicho desarrollo suele ocurrir a través de procesos inconscientes y por decisiones tomadas de manera consciente.

\section{CONCLUSIONES}

La investigación aplicada por parte de enfermería ginecoobstétrica en poblaciones privadas de libertad es casi nula no solo en el país, sino en el mundo, máxime si se trata con población masculina. En el caso del Centro 


\section{Revista Electrónica Enfermería Actual en costa Rica}

Penitenciario CAI la atención en salud no es suficiente para la cantidad de privados de libertad que habitan (sobrepoblación de casi un 50\%), ello implica un deterioro de las condiciones físicas y de salud de estas personas, así como de su salud sexual y reproductiva.

Algunas cualidades detectadas fue el temor a la infidelidad por parte su pareja, no siempre utilizan los métodos anticonceptivos a pesar de que esa omisión incrementa el riesgo de contraer ETS y de que se den embarazos no deseados. A pesar de que los privados de libertad se declaran heterosexuales, existen divergencias en sus respuestas, dado que algunas contradicen el modelo heteronormativo; tal contrariedad se relaciona con la homofobia y una sexualidad no lineal.

\section{REFERENCIAS}

Barrantes, R. (2000). Investigación un camino al conocimiento, un enfoque cualitativo y cuantitativo. Costa Rica: EUNED.

Briceño, G., Chacón, E. (2001). El género también es asunto de hombres. Serie de cuadernos de trabajo. Hacia la Equidad. San José, Costa Rica.

Campos, A., y Salas, J.M. (2002) El placer de la vida. Primera edición. San José, Costa Rica: Editorial Lara Segura.

CANTERA. (2001). El Significado de ser Hombre: Guía metodológica para el trabajo de género con hombres (Masculinidad). Nicaragua.

Carcedo, A y Sagot, M (2002) Femicidio en Costa Rica 1990-1999. INAMU. San José, Costa Rica.

Dirección General de Adaptación Social; Instituto Nacional de Criminología; Departamento de Investigación y Estadística (2007) Anuario Estadístico. Recuperado en www.mj.go.cr/Archivos\%20PDF/Estadísticas/anuario2007.pdf

ENGENDER HEALTH. (2002). Introducción a los servicios de salud reproductiva de los hombres. Nueva York, Estado Unidos. http://www.engenderhealth.org/files/pubs/gender/mrhc-1/mrh_participants_hndbk.pdf

Hard, E., Jiménez, A. (2001) Masculinidad y Género. Revista Cubana de Salud Pública. 27 (2), 77-88 (en línea). Recuperado en http://redalyc.uaemex.mx/redalyc/svc/inicio/ArtPdfRedjsp?!Cve:21427201

Guezmez, A. (2004). Reforma del Sector Salud y Derechos Sexuales y Reproductivos. Washington. OPS

Hernández, R., Fernández, C., Baptista, M. (2007). Metodología de la Investigación. México: McGraw-Hill Interamericana.

Leiva, V. (2010) Módulo Autoinstructcional: Investigación Cuantitativa. Costa Rica: Editorial Universo.

OMS (s.f.) La Sexualidad según la OMS. Recuperado en http://www.formacionintegral.com.ar/index. php?option $=$ com $\quad$ content $\&$ view $=$ article \&catid $=7 \% 3$ Asexualidad-y-educacionsexual\&id=19\%3Aoms-que-es-la-sexualidad \&Itemid=3

OMS (2008) Diez datos sobre las infecciones de transmisión sexual. Recuperado en http://www.who.int/features/factfiles/sexually transmitted_diseases/facts/es/index3.html 


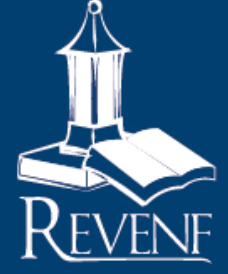

OPS/OMS y Asociación mundial de Sexología (2000) Promoción de la salud sexual: Recomendaciones para la acción. Antigua, Guatemala. Recuperado en http://www.sus.org.uy/images/96/salud_sexual_oms_ops_was.pdf

Reglamento Técnico del Sistema Penitenciario. Recuperado en

http://ministeriopublico.poder-judicial.go.cr/coop-intern/Normativa\%20Nacional/11-

Ejecucion $\% 20 \mathrm{de} \% 201 \mathrm{a} \% 20 \mathrm{Pena} / 06 . \mathrm{pdf}$

The Lancet (1994). Resumen ejecutivo de la Serie sobre salud sexual y reproductiva de Lancet. Recuperado en http://www.who.int/reproductivehealth/publications/general/srh_lancetseries_es.pdf

Vargas, Z. (2009). La investigación aplicada: una forma de conocer las realidades de la evidencia científica. Revista Educación 33 (1), 155-165. Universidad de Costa Rica. 